\title{
Aspects of Prescribing Ricci Curvature
}

\author{
Dennis DeTurck and Hubert Goldschmidt
}

\section{Dedicated to Professor M. Kuranishi on his 70th birthday}

\section{$\S 1$. Introduction}

This article concerns two problems involving the Ricci curvature of a Riemannian metric. In each of these problems, one seeks a metric whose Ricci curvature is prescribed in advance in some manner.

Let $X$ be a manifold of dimension $n \geq 3$, whose tangent and cotangent bundles we denote by $T$ and $T^{*}$, respectively. By $\otimes^{m} E, \bigwedge^{k} E$ and $S^{l} E$, we shall mean the $m$-th tensor power, $k$-th exterior product and the $l$-th symmetric product of a vector bundle $E$ over $X$, respectively. Under the natural identification of $\operatorname{Hom}\left(T, T^{*}\right)$ with $T^{*} \otimes T^{*}$, we can view a symmetric 2 -form $R$ on $X$, that is, a section of $S^{2} T^{*}$, as a morphism $R^{\mathrm{b}}: T \rightarrow T^{*}$; we shall consider the section $\operatorname{det} R$ of the line bundle Hom $\left(\bigwedge^{n} T, \bigwedge^{n} T^{*}\right)$ which is induced by $R^{b}$.

The first problem consists in finding a Riemannian metric with prescribed Ricci tensor. We are given a section $R$ of $S^{2} T^{*}$ over $X$ and we seek a Riemannian metric $g$ in some neighborhood of a given point $x_{0} \in X$ whose Ricci tensor $\operatorname{Ric}(g)$ is equal to $R$ throughout this neighborhood. The first definitive results concerning the problem of prescribing the Ricci tensor were obtained in [4]. There it was shown that, if $R\left(x_{0}\right)$ is a non-degenerate symmetric quadratic form on $T_{x_{0}}$, then a solution of this problem always exists. Examples were also given showing that, when $R\left(x_{0}\right)$ is degenerate, a solution may or may not exist. In the present paper, our attention focuses on the problem of solving the equation $\operatorname{Ric}(g)=R$ when $R$ is degenerate at every point of $X$, but has constant rank.

The second problem we consider here is the prescription of the principal Ricci curvatures of a Riemannian metric (without any prescription

Received April 20, 1996

The first author was supported in part by NSF Grant DMS 95-05175 and the second author was supported in part by NSF Grant DMS 92-03974. 
of the directions in which these principal curvatures are attained). If $\left\{\lambda_{1}, \ldots, \lambda_{n}\right\}$ are given real-valued functions on $X$, we seek a metric $g$ in a neighborhood of $x_{0} \in X$ satisfying

$$
\operatorname{det}\left(\operatorname{Ric}(g)-\lambda_{i} g\right)=0, \quad i=1, \ldots, n,
$$

on this neighborhood. If these equations hold, then these functions $\left\{\lambda_{1}, \ldots, \lambda_{n}\right\}$ are principal Ricci curvatures of $g$, i. e. if $x$ is an arbitrary point of this neighborhood, the scalars $\lambda_{1}(x), \ldots, \lambda_{n}(x)$ are eigenvalues of $\operatorname{Ric}(g)$ with respect to $g$ at $x$. This study of the principal Ricci curvatures in this sense has been proposed by many authors (in particular $[3$, p. 315] and [1, p. 180]). The reason for their interest in this question arises from the fact that, for generic Riemannian metrics, the principal Ricci curvatures provide canonical coordinates in which to express the metric. Such coordinates enable one to determine whether two Riemannian manifolds are locally (or even globally) isometric. In other words, the principal Ricci curvatures provide the key to obtaining a complete system of scalar invariants for a Riemannian manifold. Thus, it becomes desirable to know whether these important scalar invariants can be prescribed in advance. We will consider this problem in the generic case where the values $\left\{\lambda_{1}\left(x_{0}\right), \ldots, \lambda_{n}\left(x_{0}\right)\right\}$ are all distinct.

Both of our problems manifest themselves as systems of second-order partial differential equations for the metric $g$. The system corresponding to the second problem is underdetermined; in fact, it consists of $n$ equations for the $n(n+1) / 2$ unknown components of $g$. Thus we are able to solve the second problem using relatively "soft" techniques. Our main result is the following:

Theorem 1. Let $\left\{\lambda_{1}, \ldots, \lambda_{n}\right\}$ be real-analytic real-valued functions on a real-analytic manifold $X$ of dimension $n \geq 3$. Suppose that the values $\left\{\lambda_{1}\left(x_{0}\right), \ldots, \lambda_{n}\left(x_{0}\right)\right\}$ of these functions at a point $x_{0} \in X$ are distinct. Then there exists a real-analytic Riemannian metric $g$ on a neighborhood of $x_{0}$ whose principal Ricci curvatures are $\left\{\lambda_{1}, \ldots, \lambda_{n}\right\}$.

In fact, under a slightly stronger hypothesis, the above theorem admits an elementary proof, which is given in $\S 3$; our precise result can be formulated as follows:

Theorem 2. Let $\left\{\lambda_{1}, \ldots, \lambda_{n}\right\}$ be a set of $C^{\infty}$-real-valued functions on $X$ whose values $\left\{\lambda_{1}\left(x_{0}\right), \ldots, \lambda_{n}\left(x_{0}\right)\right\}$ at a point $x_{0} \in X$ are distinct. If the differentials $\left\{d \lambda_{1}, \ldots, d \lambda_{n}\right\}$ of these functions are linearly independent at the point $x_{0}$, then there exists a $C^{\infty}$-Riemannian metric $g$ defined in a neighborhood of $x_{0}$ whose principal Ricci curvatures are $\left\{\lambda_{1}, \ldots, \lambda_{n}\right\}$. 
The proof of Theorem 1 (in the analytic case) relies on our finding a non-characteristic direction for the system of equations at each generic 2 -jet of a Riemannian metric. This is in some ways similar to what happened for the non-degenerate case of our first problem in [4].

Our other problem, in which we wish to prescribe a degenerate Ricci tensor $R$, involves some fairly delicate analysis. We will work in the real-analytic category and attempt to construct a power series solution centered at $x_{0} \in X$. As is well-known, the second-order equation $\operatorname{Ric}(g)=R$ always implies an additional system of first- order (in both $g$ and $R$ ) equations. This is the so-called Bianchi identity for the Ricci curvature. In [4], it was shown that, in the non-degenerate case, the Bianchi identity is the only obstruction to the construction of the construction of the power series solution. When one wishes to extend a 2 -jet of a solution $g$ to the equation $\operatorname{Ric}(g)=R$ to a 3-jet of a solution, the Bianchi identity imposes a condition on the 1-jet of $g$. More generally, the $k$-jet of the Bianchi identity must be taken into account when specifying the $(k+1)$-jet of a solution to the equation $\operatorname{Ric}(g)=R$ in order to be able to extend this solution to one of order $k+2$. In $\S 4$, we explain how it is possible to overcome these obstructions.

However, when $R$ is degenerate and but still has constant rank, additional constraints must be placed upon the unknown metric $g$, beyond those usually implied by the Bianchi identity. In particular, conditions must be imposed on the 0-jet of $g$. These make it more difficult (and in some cases, impossible) to satisfy the higher-order prolongations of the equation $\operatorname{Ric}(g)=R$. We denote by $K$ the kernel of the morphism $R^{b}: T \rightarrow T^{*}$. In this paper, as in [6], we analyze the case where the distribution determined by the sub-bundle $K$ of $T$ is integrable and give a sufficient condition for local solvability of our equation. We are now in the midst of studying the case when this distribution is not integrable.

Our analysis of this degenerate case leads us to associate to each vector $\xi$ of the kernel $K_{x}$ of $R^{b}(x)$, with $x \in X$, a quadratic form $Q_{\xi}$ on the tangent space $T_{x}$, which depends only on $R$ and $\xi$. The obstruction to the local solvability of the equation $\operatorname{Ric}(g)=R$ can then be formulated as follows: At every point of $X$, the trace of each of these forms $Q_{\xi}$, with respect to a solution $g$ of the equation $\operatorname{Ric}(g)=R$, must vanish. The first positive result we obtained (see [6, Theorem 6.1]) states that, if all these quadratic forms vanish at every point of $X$, then the equation $\operatorname{Ric}(g)=R$ admits local solutions in the real-analytic category; this includes the case when $R$ is non-degenerate. More generally, we will also assume the space of quadratic forms $\mathcal{Q}_{x}=\left\{Q_{\xi}\right\}$, with $\xi \in K_{x}$, associated to a point $x \in X$ has constant dimension $m$, independent of the point $x$. For $x \in X$, the null-space of the pencil $\mathcal{Q}_{x}$ of quadratic 
forms contains $K_{x}$, and so its dimension $\nu(x)$ is $\geq m$.

Let $\left(x^{1}, \ldots, x^{n}\right)$ be a local coordinate system on a neighborhood $U$ of $x \in X$ such that $\left\{\partial / \partial x^{1}, \ldots, \partial / \partial x^{r}\right\}$ is a frame for the integrable subbundle $K$ of $T$. In terms of this coordinate system, we have $R_{i j}=0$, for $1 \leq i \leq n$ and $1 \leq j \leq r$; the quadratic form $Q^{k}$ corresponding to the section $\partial / \partial x^{k}$ of $K$ over $U$, with $1 \leq k \leq r$, is given by

$$
Q_{i j}^{k}=-\frac{1}{2} \frac{\partial R_{i j}}{\partial x^{k}},
$$

for $1 \leq i, j \leq n$. According to our assumption, at every point $x$ of $U$, the vector space of quadratic forms on $T_{x}$ generated by $\left\{Q^{1}(x), \ldots, Q^{r}(x)\right\}$ is $m$-dimensional.

If $m=0$, then on the open set $U$ the tensor $R$ depends only on the variables $x^{r+1}, \ldots, x^{n}$. Thus, $R$ respects the local product structure induced on $X$ by the leaves of $K$. In this case, if $n-r \geq 3$, or if $n-r=2$ and $R$ is semi-definite, then the results of [4] can be used to prove the local existence of a product metric which satisfies $\operatorname{Ric}(g)=R$. However, as indicated by corank-one examples on certain unimodular Lie groups (see [6] and [11]), there may be other solutions which do not respect the local product structure induced by $R$ on $X$.

Our most general result for this problem can be stated as follows:

Theorem 3. Let $R$ be a real-analytic symmetric 2-form on a realanalytic manifold $X$ of dimension $n \geq 3$. Suppose that the kernel $K$ of $R^{b}$ is an integrable sub-bundle of $T$ and that, for all $x \in M$, the space $\mathcal{Q}_{x}$ of quadratic forms $\left\{Q_{\xi}\right\}$, with $\xi \in K_{x}$, has constant dimension $m$. Let $x_{0} \in X$ and suppose that there exists a Riemannian metric $g_{0}$ on $X$ such that the trace (with respect to $g_{0}$ ) of the form $Q_{\xi}$ vanishes, for all $\xi \in K_{x_{0}}$. Suppose that the following conditions do not hold:

(i) we have $m=2$ and $n=4$;

(ii) we have $m=2, n=5$ and $\nu\left(x_{0}\right)=3$;

(iii) we have $m=3$ and $n=6$.

Then there exists a real-analytic Riemannian metric $g$ solution of the equation $\operatorname{Ric}(g)=R$ on a neighborhood of $x_{0}$.

This theorem tells us that, when the dimension of $X$ is $\geq 7$ and $K$ is an integrable sub-bundle of $T$, our condition for local solvability is always sufficient. When the dimension of the spaces $\mathcal{Q}_{x}$ is either zero or one, then our proof is somewhat less complicated than in the case $m \geq 2$; the cases $m=2$ or 3 require special attention.

In the special case when the kernel $K$ of $R^{b}$ is a line bundle, the obstruction to local solvability described above and Theorem 3 lead to 
a condition, which is essentially necessary and sufficient, given by the following result:

Theorem 4. Let $R$ be a real-analytic symmetric 2 -form on a realanalytic manifold $X$ of dimension $n \geq 3$. Suppose that the kernel $K$ of $R^{b}$ is a sub-bundle of $T$ of rank 1. Let $x \in M$ and $\xi \in K_{x}$. If $Q_{\xi}$ is non-zero, there exists a Riemannian metric $g$ solution of the equation $\operatorname{Ric}(g)=R$ on a neighborhood of $x$ if and only if $Q_{\xi}$ is not semi-definite.

The necessity of the condition is obvious, since $Q_{\xi}$ must ultimately be traceless with respect to the metric $g$. We present an outline of the proof of these last two theorems in $\S 4$. For all the details, we refer the reader to $[6]$.

In this paper, we shall use the theory of overdetermined partial differential equations of [9] or [2, Chapter IX] and the the notation and terminology introduced there.

We wish to thank R. Bryant and S. S. Chern for organizing a conference in March 1994 at the Mathematical Sciences Research Institute in Berkeley, where a preliminary version of Theorems 3 and 4 were presented. We thank M. Berger for suggesting the problem that led us to Theorem 1. Finally, we wish to thank M. Namba, K. Yamaguchi and the other organizers of the conference in honor of M. Kuranishi, for which this paper was written.

\section{$\S 2 . \quad$ The Ricci and Bianchi operators}

If $E$ is a fibered manifold over $X$, we denote by $J_{k}(E)$ the fibered manifold of $k$-jets of sections of $E$, and by $\pi_{k}: J_{k+l}(E) \rightarrow J_{k}(E)$ the natural projection. If $s$ is a section of $E$ over a neighborhood of $x \in X$, then $j_{k}(s)(x)$ is the $k$-jet of $s$ at $x$; the projection $\pi: J_{k}(E) \rightarrow X$ sends $j_{k}(s)(x)$ into $x$. We identify $J_{0}(E)$ with $E$. If $E$ is a vector bundle over $X$; we denote by $\mathcal{E}$ the sheaf of sections of $E$ over $X$ and we recall that there is a monomorphism of vector bundles

$$
\varepsilon: S^{k} T^{*} \otimes E \rightarrow J_{k}(E)
$$

if $s$ is a section of $E$ over a neighborhood of $x \in X$ whose $(k-1)$-jet vanishes at $x$, then $j_{k}(s)(x)$ is equal to the image under $\varepsilon$ of a unique element $\varepsilon^{-1} j_{k}(s)(x)$ of $S^{k} T^{*} \otimes E$. If $F$ is another vector bundle over $X$ and

$$
\varphi: S^{2} T^{*} \otimes E \rightarrow F
$$

is a morphism of vector bundles, we denote by

$$
\varphi^{(l)}: S^{k+l} T^{*} \otimes E \rightarrow S^{l} T^{*} \otimes F
$$


the $l$-th prolongation of $\varphi$. If $R$ is a non-degenerate section of $S^{2} T^{*}$, we consider the morphism $R^{\sharp}: T^{*} \rightarrow T$ which is the inverse of $R^{b}: T \rightarrow T^{*}$.

Let $g$ be a Riemannian metric on $X$ whose Levi-Civita connection and Ricci curvature we denote by $\nabla^{g}$ and $\operatorname{Ric}(g)$, respectively. We consider the inner product $\langle,\rangle_{g}$ on $\otimes^{k} T^{*}$ determined by $g$; then the trace, with respect to $g$, of an element $h$ of $S^{2} T^{*}$ is equal to $\operatorname{Tr}_{g} h=\langle h, g\rangle_{g}$. Let $\left(x^{1}, \ldots, x^{n}\right)$ be a coordinate system on an open subset $U$ of $X$. In expressions written in terms of such a local coordinate system, we shall use the summation convention. On $U$, the Levi-Civita connection $\nabla^{g}$ of $g$ is determined by its Christoffel symbols

$$
\Gamma_{j k}^{i}=\frac{1}{2} g^{i s}\left(\frac{\partial g_{s j}}{\partial x^{k}}+\frac{\partial g_{s k}}{\partial x^{j}}-\frac{\partial g_{j k}}{\partial x^{s}}\right),
$$

while the components of the Ricci tensor $\operatorname{Ric}(g)$ are given by

$$
\operatorname{Ric}(g)_{i j}=\frac{\partial \Gamma_{i j}^{s}}{\partial x^{s}}-\frac{\partial \Gamma_{i s}^{s}}{\partial x^{j}}+\Gamma_{i j}^{s} \Gamma_{s t}^{t}-\Gamma_{i t}^{s} \Gamma_{s j}^{t} .
$$

The Bianchi operator

$$
B_{g}: S^{2} \mathcal{T}^{*} \rightarrow \mathcal{T}^{*}
$$

is the first-order linear differential operator defined by

$$
\left(B_{g} h\right)(\xi)=\sum_{i=1}^{n}\left(\left(\nabla^{g} h\right)\left(t_{i}, \xi, t_{i}\right)-\frac{1}{2}\left(\nabla^{g} h\right)\left(\xi, t_{i}, t_{i}\right)\right),
$$

for a section $h$ of $S^{2} T^{*}$ over $X$ and $\xi \in T_{x}$, with $x \in X$, where $\left\{t_{1}, \ldots, t_{n}\right\}$ is an orthonormal basis of $T_{x}$. We also write $B_{g} h=\operatorname{Bian}(g, h)$; in fact, on $U$ we have

$$
B_{g} h=g^{i k}\left(\frac{\partial h_{i j}}{\partial x^{k}}-\frac{1}{2} \frac{\partial h_{i k}}{\partial x^{j}}-\Gamma_{i k}^{l} h_{l j}\right) d x^{j} .
$$

The symbol

$$
\sigma\left(B_{g}\right): T^{*} \otimes S^{2} T^{*} \rightarrow T^{*}
$$

of the differential operator $B_{g}$ is given by

$$
\left(\sigma\left(B_{g}\right) u\right)(\xi)=\sum_{i=1}^{n}\left(u\left(t_{i}, t_{i}, \xi\right)-\frac{1}{2} u\left(\xi, t_{i}, t_{i}\right)\right)
$$

for $\xi \in T_{x}$, where $\left\{t_{1}, \ldots, t_{n}\right\}$ is an orthonormal basis of $T_{x}$; we denote by $\sigma_{l}\left(B_{g}\right): S^{l+1} T^{*} \otimes S^{2} T^{*} \rightarrow S^{l} T^{*} \otimes T^{*}$ the $l$-th prolongation of $\sigma\left(B_{g}\right)$. 
The Ricci curvature of $g$ satisfies the Bianchi identity

$$
B_{g} \operatorname{Ric}(g)=0 \text {. }
$$

Let $S_{+}^{2} T^{*}$ be the open fibered submanifold of $S^{2} T^{*}$ consisting of the positive-definite symmetric 2-forms on $X$. Let $E$ and $F$ be vector bundles over $X$ and let $\psi: E \rightarrow F$ be a morphism of vector bundles over $J_{k}\left(S_{+}^{2} T^{*}\right)$. If $g$ is a Riemannian metric on $X$, we consider the morphism of vector bundles $\psi_{g}: E \rightarrow F$ over $X$ sending $u \in E_{x}$ into $\psi\left(j_{k}(g)(x), u\right)$, where $x \in X$.

The morphism of fibered manifolds

$$
\varphi: J_{2}\left(S_{+}^{2} T^{*}\right) \rightarrow S^{2} T^{*}
$$

sending the 2-jet $j_{2}(g)(x)$ at $x \in X$ of a Riemannian metric $g$ on $X$ into $\operatorname{Ric}(g)(x)$, is quasi-linear in the sense of [2, Chapter IX]. The symbol

$$
\sigma(\varphi): S^{2} T^{*} \otimes S^{2} T^{*} \rightarrow S^{2} T^{*}
$$

of $\varphi$ is the morphism of vector bundles over $J_{1}\left(S_{+}^{2} T^{*}\right)$ satisfying

$$
\varphi(p+\varepsilon u)=\varphi(p)+\sigma(\varphi)_{\pi_{1} p} u
$$

for all $p \in J_{2}\left(S_{+}^{2} T^{*}\right), u \in S^{2} T^{*} \otimes S^{2} T^{*}$. If $g$ is a Riemannian metric on $X$, the morphism of vector bundles

$$
\sigma(\varphi)_{g}: S^{2} T^{*} \otimes S^{2} T^{*} \rightarrow S^{2} T^{*}
$$

over $X$ is given by

$$
\begin{array}{r}
\left(\sigma(\varphi)_{g} u\right)(\xi, \eta)=\frac{1}{2} \sum_{i=1}^{n}\left\{u\left(t_{i}, \xi, t_{i}, \eta\right)+u\left(t_{i}, \eta, t_{i}, \xi\right)\right. \\
\left.-u\left(t_{i}, t_{i}, \xi, \eta\right)-u\left(\xi, \eta, t_{i}, t_{i}\right)\right\}
\end{array}
$$

for $u \in\left(S^{2} T^{*} \otimes S^{2} T^{*}\right)_{x}$ and $\xi, \eta \in T_{x}$, where $x \in X$ and $\left\{t_{1}, \ldots, t_{n}\right\}$ is an orthonormal basis of $T_{x}$. The $l$-th prolongation

$$
p_{l}(\varphi): J_{l+2}\left(S_{+}^{2} T^{*}\right) \rightarrow J_{l}\left(S^{2} T^{*}\right)
$$

of $\varphi$ is quasi-linear; if $\sigma_{l}(\varphi)_{g}$ is the $l$-th prolongation of $\sigma(\varphi)_{g}$, then we have

$$
p_{l}(\varphi)(q+\varepsilon u)=\varphi(p)+\sigma_{l}(\varphi)_{g} u,
$$

for all $q \in J_{l+2}\left(S_{+}^{2} T^{*}\right)$ and $u \in S^{l+2} T^{*} \otimes S^{2} T^{*}$, with $\pi_{1} q=j_{1}(g)(x)$. 
We set $S^{l} T^{*}=0$ for $l<0$. Let $g$ be a Riemannian metric on $X$ and let

$$
\mu_{g}:\left(S^{l} T^{*} \otimes S^{2} T^{*}\right) \oplus\left(S^{l+1} T^{*} \otimes T^{*}\right) \rightarrow S^{l-1} T^{*} \otimes T^{*}
$$

be the morphism of vector bundles sending $u \oplus v$, with $u \in S^{l} T^{*} \otimes S^{2} T^{*}$ and $v \in S^{l+1} T^{*} \otimes T^{*}$, into $\sigma_{l-1}\left(B_{g}\right) u$.

From Lemma 3.1 of [6] (with $K=\{0\}$ ) (see also [4] and [2], Chapter IX), we obtain the following:

Lemma 1. Let $g$ be a Riemannian metric on $X$. The morphism of vector bundles $\sigma(\varphi)_{g}: S^{2} T^{*} \otimes S^{2} T^{*} \rightarrow S^{2} T^{*}$ is surjective and the sequences

$$
\begin{aligned}
S^{l+2} T^{*} & \otimes S^{2} T^{*} \stackrel{\sigma_{l}(\varphi)_{g} \oplus \sigma_{l+1}\left(B_{g}\right)}{\longrightarrow} \\
& \left(S^{l} T^{*} \otimes S^{2} T^{*}\right) \oplus\left(S^{l+1} T^{*} \otimes T^{*}\right) \stackrel{\mu_{g}}{\longrightarrow} S^{l-1} T^{*} \otimes T^{*} \rightarrow 0
\end{aligned}
$$

are exact for all $l \geq 0$.

From Lemma 1, we obtain the exact sequences

$$
S^{l+2} T^{*} \otimes S^{2} T^{*} \stackrel{\sigma_{l}(\varphi)_{g}}{\longrightarrow} S^{l} T^{*} \otimes S^{2} T^{*} \stackrel{\sigma_{l-1}\left(B_{g}\right)}{\longrightarrow} S^{l-1} T^{*} \otimes T^{*} \rightarrow 0,
$$

for all $l \geq 0$. If $\beta \in T_{x}^{*}$, with $x \in X$, we consider the morphisms

$$
\sigma_{\beta}(\varphi)_{g}: S^{2} T_{x}^{*} \rightarrow S^{2} T_{x}^{*}, \quad \sigma_{\beta}\left(B_{g}\right): S^{2} T_{x}^{*} \rightarrow T_{x}^{*}
$$

defined by

$$
\sigma_{\beta}(\varphi)_{g} h=\sigma(\varphi)_{g}(\beta \otimes \beta \otimes h), \quad \sigma_{\beta}\left(B_{g}\right)(\beta \otimes h),
$$

for $h \in S^{2} T_{x}^{*}$. According to $[8, \S 6]$, if $\beta$ is a non-zero vector of $T_{x}^{*}$, with $x \in X$, the exactness of the sequences (4) gives us the exact sequence

$$
S^{2} T_{x}^{*} \stackrel{\sigma_{\beta}(\varphi)_{g}}{\longrightarrow} S^{2} T_{x}^{*} \stackrel{\sigma_{\beta}\left(B_{g}\right)}{\longrightarrow} T_{x}^{*} \rightarrow 0 .
$$

Now let $x_{0}$ be an arbitrary point of $X$ and $\rho$ be an element of $S^{2} T_{x_{0}}^{*}$. From the surjectivity of the morphism $\sigma(\varphi)_{g}$, we infer that the equation $\operatorname{Ric}(g)\left(x_{0}\right)=\rho$ can always be solved. In fact, we may assume without loss of generality that $X=\mathbb{R}^{n}$ and that $x_{0}$ is the origin of $\mathbb{R}^{n}$. The surjectivity of the morphism $\sigma(\varphi)_{g_{0}}$ tells us that there is an element $u \in\left(S^{2} T^{*} \otimes S^{2} T^{*}\right)_{x_{0}}$ satisfying $\sigma(\varphi)_{g_{0}} u=\rho$. Then the symmetric tensor $g$ given by

$$
g_{i j}=\delta_{i j}+\frac{1}{2} u_{i j, k l} x^{k} x^{l}
$$


is a Riemannian metric on a neighborhood of $x_{0}$ satisfying the desired condition.

It is obvious how to use this fact for the prescribed Ricci tensor problem; for our eigenvalue problem, in Lemma 2 below we shall choose $\rho$ to be diagonal with respect to the coordinate system so that our metric will have the desired principal Ricci curvatures at one point.

We now turn to the relation of the Bianchi identity to our problems. Let $R$ be a given section of $S^{2} T^{*}$. The morphism of fibered manifolds

$$
\psi_{R}: J_{1}\left(S_{+}^{2} T^{*}\right) \rightarrow T^{*},
$$

sending the 2-jet $j_{2}(g)(x)$ at $x \in X$ of a Riemannian metric $g$ on $X$ into $\operatorname{Bian}(g, R)(x)$, is quasi-linear. The symbol

$$
\sigma\left(\psi_{R}\right): T^{*} \otimes S^{2} T^{*} \rightarrow T^{*}
$$

of $\psi_{R}$ is a morphism of vector bundles over $S_{+}^{2} T^{*}$ which satisfies

$$
\psi_{R}(p+\varepsilon u)=\psi_{R}(p)+\sigma\left(\psi_{R}\right)_{\pi_{0} p} u,
$$

for all $p \in J_{1}\left(S_{+}^{2} T^{*}\right), u \in T^{*} \otimes S^{2} T^{*}$; in fact, this morphism is determined by

$$
\sigma\left(\psi_{R}\right)_{g}=-R^{\mathrm{b}} \circ g^{\sharp} \circ \sigma\left(B_{g}\right),
$$

where $g$ is a Riemannian metric on $X$.

The most direct evidence that the Bianchi identity $\operatorname{Bian}(g, R)=0$ provides us with an obstruction to finding solutions of the equation $\operatorname{Ric}(g)=R$ or solutions of the principal Ricci curvature problem arises from the exactness of the sequence (4), with $l=1$, when one attempts to solve the equation $\operatorname{Ric}(g)=R$ to first-order at a point of $X$. For the principal Ricci curvature problem, in Lemma 2 below we are able to satisfy this obstruction easily, because we may choose $R$ and $g$ simultaneously. The implications of the Bianchi identity for the prescribed Ricci curvature problem are more subtle and shall be examined in $\S 4$.

\section{§3. Prescribing the principal curvatures}

This section is devoted to the proofs of Theorems 1 and 2 of the introduction. If $g$ is a Riemannian metric on $X$ and $R$ is a section of $S^{2} T^{*}$, we denote by $\operatorname{det}_{g} R$ the real-valued function on $X$ which is equal to the determinant of the endomorphism $g^{\sharp} \circ R^{\mathrm{b}}$ of $T$. Then we easily see that the section $\operatorname{det} R$ of the line bundle $\operatorname{Hom}\left(\bigwedge^{n} T, \bigwedge^{n} T^{*}\right)$ vanishes at $x \in X$ if and only if the function $\operatorname{det}_{g} R$ vanishes at $x$. In 
particular, we know that $\lambda \in \mathbb{R}$ is an eigenvalue of $\operatorname{Ric}(g)$ with respect to $g$ at $x$ if and only if the function $\operatorname{det}_{g}(\operatorname{Ric}(g)-\lambda g)$ vanishes at $x \in X$, or equivalently if $\operatorname{det}(\operatorname{Ric}(g)-\lambda g)(x)=0$.

We shall require the following lemma in our proof of Theorem 2.

Lemma 2. Let $g_{0}$ be a Riemannian metric on $X$. Let $\lambda_{1}, \ldots, \lambda_{n}$ be given distinct real numbers. Then there exists a Riemannian metric $g$ on a neighborhood $U$ of $x_{0} \in X$, with $g\left(x_{0}\right)=g_{0}\left(x_{0}\right)$, such that the eigenvalues $\mu_{1}, \ldots, \mu_{n}$ of $\operatorname{Ric}(g)$ with respect to $g$ are $C^{\infty}$-functions on $U$ whose differentials are linearly independent at $x_{0}$ and which satisfy $\mu_{j}\left(x_{0}\right)=\lambda_{j}$, for $1 \leq j \leq n$.

Proof. We may assume without loss of generality that $X=\mathbb{R}^{n}$, that $x_{0}$ is the origin of $\mathbb{R}^{n}$ and that

$$
g_{0, i j}\left(x_{0}\right)=\delta_{i j}
$$

Consider the section $\rho$ of $S^{2} T^{*}$ determined by

$$
\rho_{i j}=\lambda_{j} \delta_{i j}+\left(n \delta_{i j}-1\right)\left(x_{i}+x_{j}\right) .
$$

As we have seen in $\S 2$, there is an element $u \in\left(S^{2} T^{*} \otimes S^{2} T^{*}\right)_{x_{0}}$ satisfying $\sigma(\varphi)_{g_{0}} u=\rho\left(x_{0}\right)$ and the symmetric tensor $g$ given by

$$
g_{i j}=\delta_{i j}+\frac{1}{2} u_{i j, k l} x^{k} x^{l}
$$

is a Riemannian metric on a neighborhood $U$ of $x_{0}$ which satisfies

$$
\operatorname{Ric}(g)\left(x_{0}\right)=\rho\left(x_{0}\right) .
$$

Clearly $\lambda_{1}, \ldots, \lambda_{n}$ are the eigenvalues of $\rho\left(x_{0}\right)$ with respect to the metric $g\left(x_{0}\right)=g_{0}\left(x_{0}\right)$. Since these scalars are distinct, there are $C^{\infty}$-functions $\tilde{\lambda}_{1}, \ldots, \tilde{\lambda}_{n}$ and an orthonormal frame $\left\{\xi_{1}, \ldots, \xi_{n}\right\}$ for $T$, with respect to the metric $g$, over an neighborhood $U^{\prime} \subset U$ of $x_{0}$ such that $\tilde{\lambda}_{j}\left(x_{0}\right)=\lambda_{j}$ and

$$
\rho\left(\xi_{j}, \eta\right)=\tilde{\lambda}_{j} g\left(\xi_{j}, \eta\right)
$$

for all vector fields $\eta$ on $U^{\prime}$ and $1 \leq j \leq n$. Let $\zeta$ be a vector field on $U^{\prime}$. From relation (7), with $\eta=\xi_{j}$ and $1 \leq j \leq n$, we obtain

$$
\left(\nabla_{\zeta}^{g} \rho\right)\left(\xi_{j}, \xi_{j}\right)+2 \rho\left(\xi_{j}, \nabla_{\zeta}^{g} \xi_{j}\right)=\left\langle\zeta, d \tilde{\lambda}_{j}\right\rangle+\tilde{\lambda}_{j} g\left(\xi_{j}, \nabla_{\zeta}^{g} \xi_{j}\right)
$$

Since $\xi_{j}$ is a unitary vector field, we see that $g\left(\xi_{j}, \nabla_{\zeta}^{g} \xi_{j}\right)=0$ and so by (7) we also have

$$
\rho\left(\xi_{j}, \nabla_{\zeta}^{g} \xi_{j}\right)=\tilde{\lambda}_{j} g\left(\xi_{j}, \nabla_{\zeta}^{g} \xi_{j}\right)=0 .
$$


Hence the equality (8) gives us

$$
\left(\nabla_{\zeta}^{g} \rho\right)\left(\xi_{j}, \xi_{j}\right)=\left\langle\zeta, d \tilde{\lambda}_{j}\right\rangle
$$

Since $\xi_{k}=\partial / \partial x^{k}$ at $x_{0}$ and $\Gamma_{i j}^{k}\left(x_{0}\right)=0$ for the metric $g$, from the definition of $\rho$ we see that

$$
\left(\nabla_{\xi_{k}}^{g} \rho\right)\left(\xi_{i}, \xi_{j}\right)\left(x_{0}\right)=\frac{\partial \rho_{i j}}{\partial x^{k}}\left(x_{0}\right)=\left(n-\delta_{i j}\right)\left(\delta_{i k}+\delta_{j k}\right)
$$

for $1 \leq i, j, k \leq n$. Thus we have $d \tilde{\lambda}_{j}=2(n-1) d x^{j}$ at $x_{0}$, and so the differentials of the eigenvalues $\tilde{\lambda}_{j}$ are linearly independent at $x_{0}$. Using (1) and (2), it is easily verified that the section $h=\operatorname{Ric}(g)-\rho$, which vanishes at $x_{0}$, satisfies the Bianchi identity $B_{g} h=0$ at $x_{0}$. Thus we know that

$$
\sigma\left(B_{g}\right) \varepsilon^{-1} j_{1}(h)\left(x_{0}\right)=0,
$$

and so by the exactness of the sequence (4), with $l=1$, there is an element $v$ of $\left(S^{3} T^{*} \otimes S^{2} T^{*}\right)_{x_{0}}$ satisfying

$$
\sigma_{1}(\varphi)_{g} v=-\varepsilon^{-1} j_{1}(h)\left(x_{0}\right) .
$$

Then a Riemannian metric $\tilde{g}$ on a neighborhood of $x_{0}$, whose 3 -jet at $x_{0}$ is equal to $j_{3}(g)\left(x_{0}\right)+\varepsilon v$, satisfies the relations

$$
j_{2}(\tilde{g})\left(x_{0}\right)=j_{2}(g)\left(x_{0}\right), \quad j_{1}(\operatorname{Ric}(\tilde{g})-\rho)\left(x_{0}\right)=0 .
$$

Clearly there are $C^{\infty}$-functions $\left\{\mu_{1}, \ldots, \mu_{n}\right\}$, which are eigenvalues of $\operatorname{Ric}(\tilde{g})$ with respect the metric $\tilde{g}$, such that $\mu_{j}\left(x_{0}\right)=\lambda_{j}$, for $1 \leq j \leq n$. From the equalities (9), we infer that $j_{1}\left(\mu_{j}\right)\left(x_{0}\right)=j_{1}\left(\tilde{\lambda}_{j}\right)\left(x_{0}\right)$; hence the differentials of the functions $\left\{\mu_{1}, \ldots, \mu_{n}\right\}$ are linearly independent at $x_{0}$.

Proof of Theorem 2. Let $g_{0}$ be a given Riemannian metric on $X$ and let $g$ be a Riemannian metric on a neighborhood of $x_{0}$ satisfying the assertions of Lemma 2 with respect to the distinct real numbers $\left\{\lambda_{1}\left(x_{0}\right), \ldots, \lambda_{n}\left(x_{0}\right)\right\}$ and $g_{0}$. Since their differentials are linearly independent at $x_{0}$, the eigenvalues $\mu_{1}, \ldots, \mu_{n}$ of $\operatorname{Ric}(g)$ with respect to $g$ define a diffeomorphism $\mu=\left(\mu_{1}, \ldots, \mu_{n}\right)$ of an open neighborhood of $x_{0}$ onto an open neighborhood of $y_{0}=\left(\lambda_{1}\left(x_{0}\right), \ldots, \lambda_{n}\left(x_{0}\right)\right)$ in $\mathbb{R}^{n}$. Similarly by hypothesis, the mapping $\lambda=\left(\lambda_{1}, \ldots, \lambda_{n}\right)$ is a diffeomorphism of an open neighborhood of $x_{0}$ onto an open neighborhood of $y_{0}$ in $\mathbb{R}^{n}$. Thus $\mu^{-1} \circ \lambda$ is a local diffeomorphism $\Psi$ of $X$ defined on a neighborhood of $x_{0}$ such that $\mu \circ \Psi=\lambda$ on a neighborhood of $x_{0}$. Because

$$
\operatorname{det}\left(\Psi^{*}\left(\operatorname{Ric}(g)-\mu_{j} g\right)\right)=0
$$


and $\lambda_{j}=\Psi^{*} \mu_{j}$, we see that $\Psi^{*} g$ is a metric on a neighborhood of $x_{0}$ satisfying

$$
\operatorname{det}\left(\operatorname{Ric}\left(\Psi^{*} g\right)-\lambda_{j} \Psi^{*} g\right)=0
$$

for all $1 \leq j \leq n$.

The remainder of this section is devoted to the proof of Theorem 1 . Let $A_{n}$ be the affine space of all real monic polynomials in the variable $\lambda$ of degree $n$, which is modeled on the vector space $V_{n-1}$ of all real polynomials in the variable $\lambda$ of degree $\leq n-1$. Let $E$ and $F$ be the trivial bundles over $X$ whose fibers are equal to $A_{n}$ and $V_{n-1}$, respectively. Then $F$ is an affine bundle over $X$ modeled on the vector bundle $E$. We consider the morphism of fibered manifolds

$$
\Phi: J_{2}\left(S_{+}^{2} T^{*}\right) \rightarrow F,
$$

sending the 2-jet $j_{2}(g)(x)$ at $x \in X$ of a Riemannian metric $g$ on $X$ into the monic polynomial $(-1)^{n} \operatorname{det}_{g}(\operatorname{Ric}(g)-\lambda g)(x)$ in the variable $\lambda$. The symbol of $\Phi$ is the morphism

$$
\sigma(\Phi): S^{2} T^{*} \otimes S^{2} T^{*} \rightarrow E
$$

of vector bundles over $J_{2}\left(S_{+}^{2} T^{*}\right)$ defined as follows. If $g$ is a Riemannian metric on $X$ and $h$ is a section of $S^{2} T^{*}$ over a neighborhood of $x \in X$ satisfying $j_{1}(h)(x)=0$, then $g+t h$ is a Riemannian metric on neighborhood of $x$ for $|t|<\epsilon$, with $\epsilon>0$; the morphism $\sigma(\Phi)_{g}$ sends the element $\varepsilon^{-1} j_{2}(h)(x)$ of $\left(S^{2} T^{*} \otimes S^{2} T^{*}\right)_{x}$ into the vector $\frac{d}{d t} \Phi(g+t h)_{\mid t=0}$ of $E_{x}$.

Let $g$ be a Riemannian metric on $X$ and $x \in X$. Let $\left\{\lambda_{1}, \ldots, \lambda_{n}\right\}$ be the eigenvalues of $\operatorname{Ric}(g)$ with respect to $g$ at $x$; then there is an orthonormal basis $\left\{\xi_{1}, \ldots, \xi_{n}\right\}$ of $T_{x}$, with respect to the metric $g$, consisting of eigenvectors for $\operatorname{Ric}(g)$ satisfying

$$
g^{\sharp} \cdot \operatorname{Ric}(g)^{b} \xi_{j}=\lambda_{j} \xi_{j},
$$

for $1 \leq j \leq n$. Let $\operatorname{Ric}_{g}^{\prime}: S^{2} \mathcal{T}^{*} \rightarrow S^{2} \mathcal{T}^{*}$ be the linear differential operator of order 2 which is the linearization along $g$ of the non-linear operator $h \mapsto \operatorname{Ric}(h)$, where $h$ is a Riemannian metric on $X$. Let $h$ be a section of $S^{2} T^{*}$ over $X$. For $|t|<\epsilon$, we know that $g_{t}=g+t h$ is a Riemannian metric on a neighborhood of $x$; by definition, we have

$$
\operatorname{Ric}_{g}^{\prime}(h)=\frac{d}{d t} \operatorname{Ric}_{g}(g+t h)_{\mid t=0} .
$$

For $|t|<\epsilon$ and $\lambda \in \mathbb{R}$, we consider the endomorphism

$$
B_{t}^{\lambda}=g_{t}^{\sharp} \circ \operatorname{Ric}\left(g_{t}\right)^{b}-\lambda \mathrm{id}
$$


of $T_{x}$; then it is easily seen that

$$
B=\frac{d}{d t} B_{t \mid t=0}^{\lambda}=\left(g^{\sharp} \circ \operatorname{Ric}_{g}^{\prime}(h)^{b}-g^{\sharp} \circ h^{b} \circ g^{\sharp} \circ \operatorname{Ric}(g)^{b}\right)(x) .
$$

Clearly the vector

$$
p(\lambda)=\frac{d}{d t} \operatorname{det}_{g_{t}}\left(\operatorname{Ric}\left(g_{t}\right)-\lambda g_{t}\right)(x)_{\mid t=0}
$$

of $V_{n-1}$ is determined by the relation

$\sum_{i=1}^{n} B_{0}^{\lambda} \xi_{1} \wedge \ldots \wedge B_{0}^{\lambda} \xi_{i-1} \wedge B \xi_{i} \wedge B_{0}^{\lambda} \xi_{i+1} \wedge \ldots \wedge B_{0}^{\lambda} \xi_{n}=p(\lambda) \xi_{1} \wedge \ldots \wedge \xi_{n}$

for $\lambda \in \mathbb{R}$. According to (10), for $1 \leq i \leq n$, we have $B_{0}^{\lambda} \xi_{i}=\left(\lambda_{i}-\lambda\right) \xi_{i}$ and we see that

$$
\begin{aligned}
g\left(B \xi_{i}, \xi_{i}\right) & =\operatorname{Ric}_{g}^{\prime}(h)\left(\xi_{i}, \xi_{i}\right)-\left\langle\xi_{i}, h^{b} \cdot g^{\sharp} \cdot \operatorname{Ric}(g)^{b} \xi_{i}\right\rangle \\
& =\operatorname{Ric}_{g}^{\prime}(h)\left(\xi_{i}, \xi_{i}\right)-\lambda_{i} h\left(\xi_{i}, \xi_{i}\right) .
\end{aligned}
$$

For $1 \leq i \leq n$, we consider the polynomial

$$
P_{i}(\lambda)=\prod_{\substack{j=1 \\ j \neq i}}^{n}\left(\lambda_{j}-\lambda\right)
$$

of $V_{n-1}$. From the previous relations, we obtain the equality

$$
p(\lambda)=\sum_{i=1}^{n}\left(\operatorname{Ric}_{g}^{\prime}(h)-\lambda_{i} h\right)\left(\xi_{i}, \xi_{i}\right) \cdot P_{i}(\lambda)
$$

Since the symbol of the differential operator $\operatorname{Ric}_{g}^{\prime}$ is equal to $\sigma(\varphi)_{g}$, from (11) we deduce that the morphism $\sigma(\Phi)_{g}$ at $x$ is given by

$$
\sigma(\Phi)_{g} u=(-1)^{n} \sum_{i=1}^{n}\left(\sigma(\varphi)_{g} u\right)\left(\xi_{i}, \xi_{i}\right) \cdot P_{i}(\lambda)
$$

for $u \in\left(S^{2} T^{*} \otimes S^{2} T^{*}\right)_{x}$. If $\beta \in T_{x}^{*}$, let

$$
\sigma_{\beta}(\Phi)_{j_{2}(g)(x)}: S^{2} T_{x}^{*} \rightarrow E_{x}
$$


be the mapping sending the element $v$ of $S^{2} T_{x}^{*}$ into $\sigma(\Phi)_{g}(\beta \otimes \beta \otimes v)$; thus we have

$$
\sigma_{\beta}(\Phi)_{j_{2}(g)(x)} v=(-1)^{n} \sum_{i=1}^{n}\left(\sigma_{\beta}(\varphi)_{g} v\right)\left(\xi_{i}, \xi_{i}\right) \cdot P_{i}(\lambda),
$$

for $v \in S^{2} T_{x}^{*}$.

Now assume that the eigenvalues $\left\{\lambda_{1}, \ldots, \lambda_{n}\right\}$ are distinct. Then the $n$ polynomials $\left\{P_{1}, \ldots, P_{n}\right\}$ of degree $n-1$ form a basis for $V_{n-1}$; this fact follows from the relations $P_{i}\left(\lambda_{i}\right) \neq 0$ and $P_{i}\left(\lambda_{j}\right)=0$ when $j \neq i$. Let $\beta$ be a non-zero vector of $T_{x}^{*}$; we write $\beta_{i}=\beta\left(\xi_{i}\right)$, for $1 \leq i \leq n$. According to formula (14), the mapping (13) is surjective if and only if the mapping $S^{2} T_{x}^{*} \rightarrow \mathbb{R}^{n}$, which sends an element $v$ of $S^{2} T_{x}^{*}$ into the $n$-tuple

$$
\left(\left(\sigma_{\beta}(\Phi)_{g} v\right)\left(\xi_{i}, \xi_{i}\right)\right)_{1 \leq i \leq n},
$$

is surjective. From the exactness of the sequence (5), we infer that the mapping (13) is surjective if and only if, given an arbitrary element $y=\left(y_{1}, \ldots, y_{n}\right) \in \mathbb{R}^{n}$, there exists $v \in S^{2} T_{x}^{*}$ satisfying $v\left(\xi_{i}, \xi_{i}\right)=y_{i}$ for all $1 \leq i \leq n$ and $\sigma_{\beta}\left(B_{g}\right) v=0$. Hence the mapping (13) is surjective if and only if the following assertion holds: for all $y=\left(y_{1}, \ldots, y_{n}\right) \in \mathbb{R}^{n}$, there exists an $n \times n$ symmetric matrix $A=\left(a_{i j}\right)_{1 \leq i, j \leq n}$ satisfying $a_{i i}=0$ and

$$
\sum_{j=1}^{n} \beta_{j} a_{i j}=\beta_{i} z_{i}
$$

where

$$
z_{i}=-y_{i}+\frac{1}{2} \sum_{j=1}^{n} y_{j}
$$

for all $1 \leq i \leq n$. If we set $m=n(n-1) / 2$, we may view the equations (15) as a system of linear equations

$$
(C A)_{i}=\beta_{i} z_{i}, \quad i=1, \ldots, n,
$$

where the matrix $A$ is viewed as a vector in $\mathbb{R}^{m}$ and $C$ is a given $n \times m$ matrix each of whose entries is equal either to 0 or to one of the $\beta_{j}$ 's. We then see that our condition for the surjectivity of the mapping (13) can be reformulated as follows: for all $z=\left(z_{1}, \ldots, z_{n}\right) \in \mathbb{R}^{n}$, we can solve the system (16) for $A \in \mathbb{R}^{m}$. If $1 \leq i, j, k \leq n$ are distinct integers, it is easily verified that the matrix $C$ possesses an $n \times n$-minor whose determinant is equal to $\pm 2 \beta_{i}^{n-2} \beta_{j} \beta_{k}$. On the other hand, if there are 
at most two non-vanishing coefficients $\beta_{j}$ of $\beta$, it is easily seen that this last condition for the surjectivity of (13) does not hold. Thus we have proved the following lemma:

Lemma 3. Let $g$ be a Riemannian metric on $X$. Assume that the eigenvalues $\left\{\lambda_{1}, \ldots, \lambda_{n}\right\}$ of $\operatorname{Ric}(g)$ with respect to $g$ at $x \in X$ are distinct. Let $\left\{\xi_{1}, \ldots, \xi_{n}\right\}$ be an orthonormal basis of $T_{x}$, with respect to $g$, consisting of eigenvectors for $\operatorname{Ric}(g)$ and satisfying the relations (10), for $1 \leq j \leq n$. If $\beta$ is a vector of $T_{x}^{*}$, then the mapping (13) is surjective if and only if there exist three distinct integers $1 \leq i, j, k \leq n$ such that $\beta\left(\xi_{i}\right) \cdot \beta\left(\xi_{j}\right) \cdot \beta\left(\xi_{k}\right) \neq 0$.

Let $N_{2}^{\prime}$ be the subset of $J_{2}\left(S_{+}^{2} T^{*}\right)$ consisting of all 2-jets $j_{2}(g)(x)$, where $x \in X$ and $g$ is a Riemannian metric on $X$ for which the eigenvalues of $\operatorname{Ric}(g)(x)$ with respect to $g(x)$ are distinct. According to Lemma 2, we see that $N_{2}^{\prime}$ is an open fibered submanifold of $J_{2}\left(S_{+}^{2} T^{*}\right)$, with $\pi_{0} N_{2}^{\prime}=S_{+}^{2} T^{*}$.

Let $\left\{\lambda_{1}, \ldots, \lambda_{n}\right\}$ be given real-valued functions on $X$; assume that, for all $x \in X$, we have $\lambda_{i}(x) \neq \lambda_{j}(x)$, for $i \neq j$. We consider the $A_{n}$-valued function

$$
P(\lambda)=\prod_{j=1}^{n}\left(\lambda-\lambda_{j}\right)
$$

on $X$ and the subset

$$
N_{2}=\left\{p \in N_{2}^{\prime} \mid \Phi(p)=P(\lambda)(x), \text { where } x=\pi(p)\right\}
$$

of $J_{2}\left(S_{+}^{2} T^{*}\right)$. Clearly, if a Riemannian metric $g$ satisfies $\Phi\left(j_{2}(g)\right)=$ $P(\lambda)$ at $x \in X$, then the eigenvalues of its Ricci tensor with respect to $g$ at $x$ are equal to $\left\{\lambda_{1}(x), \ldots, \lambda_{n}(x)\right\}$. By Lemma 2, we know that $P(X) \subset \Phi\left(N_{2}^{\prime}\right)$. If $p \in N_{2}^{\prime}$ and $x=\pi(p)$, according to Lemma 3 there exists an element $\beta$ of $T_{x}^{*}$ such that the mapping $\sigma_{\beta}(\Phi)_{p}: S^{2} T_{x}^{*} \rightarrow E_{x}$ is surjective. From Lemma 1 of [7], we obtain:

Proposition 1. Let $\left\{\lambda_{1}, \ldots, \lambda_{n}\right\}$ be given real-valued functions on $X$; assume that, for all $x \in X$, we have $\lambda_{i}(x) \neq \lambda_{j}(x)$, for $i \neq j$. Then the subset $N_{2}$ of $J_{2}\left(S_{+}^{2} T^{*}\right)$ is a formally integrable differential equation.

Thus $N_{2}$ is an underdetermined differential equation. If the manifold $X$ is real-analytic and if the functions $\left\{\lambda_{1}, \ldots, \lambda_{n}\right\}$ are real-analytic, Theorem 2.2, Chapter IX of [9] provides us with the existence of local solutions of the equation $N_{2}$; in fact, if $x \in X$, it gives us a real-analytic 
Riemannian metric $g$ satisfying $\Phi\left(j_{2}(g)\right)=P(\lambda)$ on a neighborhood of $x \in X$. This completes the proof of Theorem 1 .

\section{$\S 4 . \quad$ Prescribing the Ricci tensor}

Let $R$ be a given section of $S^{2} T^{*}$ over $X$ of constant rank. The expression $\operatorname{Bian}(g, R)$ is first-order in the metric $g$ (as well as in $R$ ); in right-hand side of (1), the highest (first) derivatives of $g$ are all multiplied by coefficients of $R$. This is reflected in formula (6) and, when $R$ is degenerate, is the source of the problems which need to be overcome. We now indicate how to solve the prescribed Ricci curvature problem and why the Bianchi identity is the only obstruction to local solvability in the non-degenerate case.

We suppose that $R$ is non-degenerate and we consider the quasilinear morphism of fibered manifolds

$$
\psi_{R}^{\prime}: J_{2}\left(S_{+}^{2} T^{*}\right) \rightarrow T^{*}
$$

determined by

$$
\psi_{R}^{\prime}\left(j_{1}(g)\right)=g^{b} \cdot R^{\sharp} \cdot \psi_{R}\left(j_{1}(g)\right),
$$

where $g$ is a Riemannian metric. According to (6) and Lemma 1, the symbol

$$
\sigma\left(\psi_{R}^{\prime}\right): T^{*} \otimes S^{2} T^{*} \rightarrow T^{*}
$$

of $\psi_{R}^{\prime}$ is the surjective morphism of vector bundles over $S_{+}^{2} T^{*}$ determined by the equality

$$
\sigma\left(\psi_{R}^{\prime}\right)_{g}=-\sigma\left(B_{g}\right) u
$$

where $g$ is a Riemannian metric on $X$. Because of (2), a solution $g$ to our original problem is also a solution of the system of second-order equations

$$
\operatorname{Ric}(g)=R, \quad j_{1}\left(\psi_{R}^{\prime}(g)\right)=0 .
$$

The exactness of the sequences (3) is the main ingredient in the local solvability of this system of equations. In fact, since the morphism $\sigma\left(\psi_{R}^{\prime}\right)$ is surjective, we obtain a Riemannian metric $g$ on a neighborhood of $x_{0} \in X$ satisfying $\psi_{R}^{\prime}(g)\left(x_{0}\right)=0$. Next, let $l \geq 0$ and let $g$ be a Riemannian metric on a neighborhood of $x_{0}$ whose $(l+1)$-jet at $x_{0}$ satisfies

$$
j_{l-1}(\operatorname{Ric}(g)-R)\left(x_{0}\right)=0, \quad j_{l}\left(\psi_{R}^{\prime}(g)\right)\left(x_{0}\right)=0
$$


Then we have $j_{l}(\operatorname{Bian}(g, R))\left(x_{0}\right)=0$. Thus if $u \in\left(S^{l} T^{*} \otimes S^{2} T^{*}\right)_{x_{0}}$ and $v \in\left(S^{l+1} T^{*} \otimes T^{*}\right)_{x_{0}}$ are the elements defined by

$$
u=\varepsilon^{-1} j_{l}(\operatorname{Ric}(g)-R)\left(x_{0}\right), \quad v=\varepsilon^{-1} j_{l+1}\left(\psi_{R}^{\prime}(g)\right)\left(x_{0}\right),
$$

by (2) we easily see that $\mu_{g}(u, v)=0$. Hence by the exactness of the sequence (3), there exists an element $w \in\left(S^{l+2} T^{*} \otimes S^{2} T^{*}\right)_{x_{0}}$ such that

$$
\sigma_{l}(\varphi)_{g} w=u, \quad \sigma_{l+1}\left(B_{g}\right) w=v
$$

Since the morphisms $\varphi$ and $\psi_{R}^{\prime}$ are quasi-linear, we see that a Riemannian metric on a neighborhood of $x_{0}$, whose $(l+2)$-jet at $x_{0}$ is equal to $j_{l+2}(g)\left(x_{0}\right)-\varepsilon w$, satisfies equations (18), with $l$ replaced by $l+1$. Thus we obtain a formal solution at $x_{0}$ of our system (17), which has the special property that its jet of order 2 at $x_{0}$ is strongly prolongable. If $R$ is a real-analytic section, a result of Malgrange [10] asserts that the equations (17) admit a convergent series solution at $x_{0}$, and yields a solution of our original equation.

We now turn to the case where $R$ is degenerate. A solution $g$ to the equation $\operatorname{Ric}(g)=R$ must satisfy some further equations which we now proceed to derive. The kernel $K$ of the morphism $R^{b}: T \rightarrow T^{*}$ is a sub-bundle of $T^{*}$. We say that this bundle $K$ is integrable if the sheaf $\mathcal{K}$ is stable under the Lie bracket.

Let $\nabla$ be an arbitrary torsionless connection in $T$. We consider the section $\nabla R$ of $T^{*} \otimes S^{2} T^{*}$ and the Lie derivative $\mathcal{L}_{\xi} R$ of $R$ along a vector field $\xi$ on $X$.

The following lemma associates a section $Q$ of $S^{2} T^{*} \otimes K^{*}$ to the section $R$ of $S^{2} T^{*}$.

Lemma 4. The section

$$
Q=(\lambda(\nabla R))_{\mid T \otimes T \otimes K}
$$

of $S^{2} T^{*} \otimes K^{*}$ is independent of the torsionless connection $\nabla$ and has the following properties:

(i) If $\xi$ is a section of $K$ over $X$, we have

$$
Q(\eta, \zeta, \xi)=-\frac{1}{2}\left(\mathcal{L}_{\xi} R\right)(\eta, \zeta)
$$

for all $\eta, \zeta \in T$.

(ii) If $\eta \in T, \xi_{1}, \xi_{2}, \zeta \in K$, we have

$$
Q\left(\eta, \xi_{1}, \xi_{2}\right)+Q\left(\eta, \xi_{2}, \xi_{1}\right)=0, \quad Q\left(\zeta, \xi_{1}, \xi_{2}\right)=0
$$


(iii) The sub-bundle $K$ of $T$ is integrable if and only if

$$
Q\left(\eta, \xi_{1}, \xi_{2}\right)=0
$$

for all $\xi_{1}, \xi_{2} \in K, \eta \in T$.

Proof. If $\nabla, \nabla^{\prime}$ are torsionless connections in $T$, then there is a section $L$ of $S^{2} T^{*} \otimes T$ such that

$$
\nabla_{\xi}^{\prime} \eta-\nabla_{\xi} \eta=L(\xi, \eta)
$$

for $\xi, \eta \in \mathcal{T}$. It is easily verified that

$$
\left(\lambda\left(\nabla^{\prime} R-\nabla R\right)\right)\left(\zeta_{1}, \zeta_{2}, \xi\right)=-R\left(L\left(\zeta_{1}, \zeta_{2}\right), \xi\right),
$$

for $\xi, \zeta_{1}, \zeta_{2} \in T$. If $\xi$ belongs to $K$, the right-hand side of the above equation vanishes, and so we see that $Q$ is independent of $\nabla$. Since $\nabla$ is torsionless, according to the definition of $Q$ we have

$$
Q(\eta, \zeta, \xi)=\frac{1}{2}(R([\xi, \eta], \zeta)+R(\eta,[\xi, \zeta])-\xi \cdot R(\eta, \zeta))=-\frac{1}{2}\left(\mathcal{L}_{\xi} R\right)(\eta, \zeta),
$$

for all $\xi \in \mathcal{K}, \eta, \zeta \in \mathcal{T}$. We thus obtain (19) and see that

$$
Q\left(\eta, \xi_{1}, \xi_{2}\right)=-\frac{1}{2} R\left(\eta,\left[\xi_{1}, \xi_{2}\right]\right),
$$

for all $\xi_{1}, \xi_{2} \in \mathcal{K}, \eta \in \mathcal{T}$. Assertions (ii) and (iii) follow directly from this equality.

If $\xi \in K$, we denote by $Q_{\xi}$ the element of $S^{2} T^{*}$ defined by

$$
Q_{\xi}(\eta, \zeta)=Q(\eta, \zeta, \xi),
$$

for $\eta, \zeta \in T$; from (20), we deduce that

$$
Q_{\xi}(\xi, \eta)=0
$$

for all $\eta \in T$. If $\xi$ is a section of $K$ over $X$, by (19) we have

$$
Q_{\xi}=-\frac{1}{2} \mathcal{L}_{\xi} R \text {. }
$$

If $Q$ vanishes identically, then by Lemma 4,(iii) we see that $K$ is integrable. Let $K^{\prime \prime}$ be the sub-bundle of $T$, with possibly varying fiber, consisting of all elements $\xi \in T$ satisfying

$$
Q(\eta, \xi, \zeta)=0,
$$

for all $\eta \in T, \zeta \in K$. If $K$ is integrable, then according to Lemma 4,(iii) we see that $K \subset K^{\prime \prime}$, and so the dimension $\nu(x)$ of $K_{x}^{\prime \prime}$ is greater the rank 
of $K$. We shall consider the morphism of vector bundles $\iota: K \rightarrow S^{2} T^{*}$, which sends $\xi \in K$ into $Q_{\xi}$.

If $g$ is a Riemannian metric on $X$, using $\nabla^{g}$ to define $Q$, we see that

$$
\left(B_{g} R\right)(\xi)=\operatorname{Tr}_{g} Q_{\xi},
$$

for all $\xi \in K$. This provides us with a new obstruction to solvability; indeed, by (2) any solution $g$ to the equation $\operatorname{Ric}(g)=R$ must also satisfy

$$
\operatorname{Tr}_{g} Q_{\xi}=0,
$$

for all $\xi \in K$. If $g$ is a Riemannian metric on $X$ and $\xi$ is a vector of $K$ satisfying the relation (23), then we easily see that either $Q_{\xi}$ vanishes or is not semi-definite. These remarks imply the following:

Theorem 5. Let $x \in X$ and $\xi \in K_{x}$. If $Q_{\xi}$ does not vanish and is semi-definite, then there does not exist a Riemannian metric $g$ on any neighborhood of $x$ such that $\operatorname{Ric}(g)=R$.

In [6, Lemma 2.3], using (21) we prove the more precise version of the previous observation:

Lemma 5. Let $x \in X$ and $\xi \in K_{x}$; assume that $Q_{\xi}$ does not vanish. Then there exists a Riemannian metric $g$ on $X$ such that

$$
\operatorname{Tr}_{g} Q_{\xi}=0
$$

if and only if $Q_{\xi}$ is not semi-definite.

By the preceding lemma, we see that Theorem 4 is a direct consequence of Theorem 3 .

In terms of a local coordinate system $\left(x^{1}, \ldots, x^{n}\right)$ on an open subset $U$ of $X$, using the flat connection $\nabla$ on $U$ satisfying $\nabla \partial / \partial x^{j}=0$, for $1 \leq j \leq n$, to compute the section $Q$, we see that the section $Q_{\xi}$ of $S^{2} T^{*}$ over $U$ corresponding to the section $\xi=\xi^{j} \partial / \partial x^{j}$ of $K$ is given by

$$
\left(Q_{\xi}\right)_{i j}=\frac{1}{2}\left(\frac{\partial R_{j k}}{\partial x^{i}}+\frac{\partial R_{i k}}{\partial x^{j}}-\frac{\partial R_{i j}}{\partial x^{k}}\right) \xi^{k}
$$

for $1 \leq i, j \leq n$. If the bundle $K$ is integrable and if $\left\{\partial / \partial x^{1}, \ldots, \partial / \partial x^{r}\right\}$ is a frame for this bundle $K$ over $U$, then we have $R_{i j}=0$, for $1 \leq i \leq n$ and $1 \leq j \leq r$, and the section $Q^{k}=Q_{\xi_{k}}$ of $S^{2} T^{*}$ over $U$ corresponding to the section $\xi_{k}=\partial / \partial x^{k}$ of $K$, with $1 \leq k \leq r$, is given by

$$
Q_{i j}^{k}=-\frac{1}{2} \frac{\partial R_{i j}}{\partial x^{k}},
$$


for $1 \leq i, j \leq n$. If the morphism of vector bundles $\iota: K \rightarrow S^{2} T^{*}$ has constant rank equal to $m$, then, at every point $x$ of $U$, the vector space of elements of $S^{2} T_{x}^{*}$ generated by $\left\{Q^{1}(x), \ldots, Q^{r}(x)\right\}$ is $m$-dimensional.

We now consider some simple examples of degenerate Ricci candidates.

Example 1. A simple example of non-existence arising from Theorem 5 is given by the following. We consider the symmetric 2-form

$$
R=d x^{2} \otimes d x^{2}+\cdots+d x^{n-1} \otimes d x^{n-1}+\left(1+x^{1}\right) d x^{n} \otimes d x^{n}
$$

on $\mathbb{R}^{n}$. Note that the rank of $R$ is equal to $n-1$ in a neighborhood $U$ of the origin and that the vector field $\xi=\partial / \partial x^{1}$ generates the sub-bundle $K$ over $U$. According to (25), for $x \in U$, the element $Q^{1}(x)$ of $S^{2} T_{x}^{*}$ is determined by $Q_{i j}^{1}(x)=\lambda_{i} \delta_{i j}$, where $\lambda_{i}=0$ for $1 \leq i \leq n-1$ and $\lambda_{n}=1$; thus $Q^{1}(x)$ is non-zero and semi-definite. Hence we know that there does not exist a Riemannian metric $g$ satisfying $\operatorname{Ric}(g)=R$ on any neighborhood of a point of $U$.

Example 2. The tensor $R= \pm d x^{3} \otimes d x^{3}$ on $\mathbb{R}^{3}$ clearly has rank one everywhere. The vector fields $\left\{\partial / \partial x^{1}, \partial / \partial x^{2}\right\}$ generate the integrable sub-bundle $K$ over $\mathbb{R}^{3}$, and by (25) the sections $Q^{1}$ and $Q^{2}$ of $S^{2} T^{*}$ both vanish. As noted in the introduction, if $x$ is an arbitrary point of $\mathbb{R}^{3}$, we can apply Theorem 3 to obtain the existence of a metric $g$ satisfying $\operatorname{Ric}(g)=R$ on a neighborhood of $x$. However, it is worth noting that although $R$ splits as a product, the same cannot be true for $g$, since then the one-dimensional factor of $g$ would have to exhibit non-zero curvature, which is impossible. It is therefore interesting to exhibit an explicit solution $g$ of the equation $\operatorname{Ric}(g)=R$. We first consider the case when $R=d x^{3} \otimes d x^{3}$ and seek a solution $g$ of the form

$$
g=f\left(x^{3}\right)^{2}\left(d x^{1} \otimes d x^{1}+d x^{2} \otimes d x^{2}\right)+h\left(x^{3}\right)^{2} d x^{3} \otimes d x^{3},
$$

where $f$ and $h$ are non-vanishing functions of $t=x^{3}$. We shall use a prime to denote the derivative with respect to $t$, and will no longer indicate the dependence of $f$ and $h$ on $t$. This metric $g$ is a warped product and is necessarily conformally flat. Its only possibly non-vanishing Christoffel symbols are determined by

$$
\Gamma_{13}^{1}=\Gamma_{23}^{2}=f^{\prime} / f, \quad \Gamma_{11}^{3}=\Gamma_{22}^{3}=-f f^{\prime} / h^{2}, \quad \Gamma_{33}^{3}=h^{\prime} / h .
$$

An elementary calculation yields the components of its Ricci tensor:

$$
\operatorname{Ric}(g)_{11}=\operatorname{Ric}(g)_{22}=-\left(\frac{f^{\prime}}{h}\right)^{2}-\frac{f}{h}\left(\frac{f^{\prime}}{h}\right)^{\prime}, \quad \operatorname{Ric}(g)_{33}=-2 \frac{h}{f}\left(\frac{f^{\prime}}{h}\right)^{\prime}
$$


and $\operatorname{Ric}(g)_{i j}=0$ for $i \neq j$. If we set $f(t)=e^{a t}$ and $h(t)=C e^{2 a t}$, where $a, C \in \mathbb{R}$ and $C>0$, we find that the Ricci tensor of the metric $g$ is equal to $2 a^{2} d x^{3} \otimes d x^{3}$. With this choice of positive functions $f$ and $h$, where $a=1 / \sqrt{2}$ and $C>0$, the metric $g$ given by (26) is a solution of the equation $\operatorname{Ric}(g)=d x^{3} \otimes d x^{3}$ on $\mathbb{R}^{3}$. Finally, the only possibly non-vanishing Christoffel symbols of the metric

$$
g^{\prime}=e^{-2 a x^{3}} d x^{1} \otimes d x^{1}+e^{2 a x^{3}} d x^{2} \otimes d x^{2}+d x^{3} \otimes d x^{3}
$$

on $\mathbb{R}^{3}$, with $a \in \mathbb{R}$, are determined by:

$$
\Gamma_{13}^{1}=-a, \quad \Gamma_{23}^{2}=a, \quad \Gamma_{11}^{3}=a e^{-2 a x^{3}}, \quad \Gamma_{22}^{3}=-a e^{2 a x^{3}} .
$$

Then one easily verifies that the Ricci tensor of the metric $g^{\prime}$ is equal to $-2 a^{2} d x^{3} \otimes d x^{3}$. Thus this metric $g^{\prime}$, with $a=1 / \sqrt{2}$, is a solution of the equation $\operatorname{Ric}\left(g^{\prime}\right)=-d x^{3} \otimes d x^{3}$ on $\mathbb{R}^{3}$. It is interesting to examine the geometry of the metric $g^{\prime}$ on $\mathbb{R}^{3}$. For $c \in \mathbb{R}$, the hypersurface of $\mathbb{R}^{3}$ defined by $x^{3}=c$ is a flat submanifold (but not complete), while the hypersurfaces of $\mathbb{R}^{3}$ defined by $x^{1}=c$ and $x^{2}=c$ have constant negative curvature (equal to $-a^{2}$ ) and are also not complete.

Example 3. The tensor

$$
R=\left(d x^{2}+x^{3} d x^{1}\right) \otimes\left(d x^{2}+x^{3} d x^{1}\right)+d x^{3} \otimes d x^{3}
$$

on $\mathbb{R}^{3}$ has rank two and the kernel $K$ is generated by the vector field $\xi=\partial / \partial x^{1}-x^{3} \partial / \partial x^{2}$. By (24), the section $Q=Q_{\xi}$ is non-zero at all points of $\mathbb{R}^{3}$; in fact, its non-zero coefficients are given by

$$
Q_{13}=\frac{x^{3}}{2}, \quad Q_{23}=\frac{1}{2} .
$$

The Euclidean metric

$$
g_{0}=d x^{1} \otimes d x^{1}+d x^{2} \otimes d x^{2}+d x^{3} \otimes d x^{3}
$$

clearly satisfies $\operatorname{Tr}_{g_{0}} Q_{\xi}=0$ on $\mathbb{R}^{3}$. Hence, if $x$ is an arbitrary point of $\mathbb{R}^{3}$, Theorem 4 gives us a metric $g$ satisfying $\operatorname{Ric}(g)=R$ on a neighborhood of $x$.

Example 4. The tensor

$$
R=\left(d x^{2}+x^{3} d x^{1}\right) \otimes\left(d x^{2}+x^{3} d x^{1}\right)
$$

on $\mathbb{R}^{3}$ has rank one and the kernel $K$ is generated by the vector fields $\xi_{1}=\partial / \partial x^{1}-x^{3} \partial / \partial x^{2}$ and $\xi_{2}=\partial / \partial x^{3}$. Since $\left[\xi_{1}, \xi_{2}\right]=\partial / \partial x^{2}$, the 
distribution determined by $K$ is not integrable. If $g$ is a Riemannian metric on $\mathbb{R}^{3}$, then by (1) we see that

$$
\left(B_{g} R\right)\left(\xi_{1}\right)=x^{3} g^{13}+g^{23}, \quad\left(B_{g} R\right)\left(\xi_{2}\right)=-\left(x^{3} g^{11}+g^{12}\right) .
$$

It is easily seen that the integrability condition $B_{g} R=0$ places two conditions of order zero on the metric $g$; namely, a solution $g$ of the equation $\operatorname{Ric}(g)=R$ must satisfy the relations

$$
g_{23}=0, \quad g_{12}=x^{3} g_{22} \text {. }
$$

This example shows that the singularity of the tensor $R$ places substantial restrictions on the 0 -jet of the solution metric $g$.

We now present an outline of the proof of Theorem 3. For simplicity, we assume that the morphism $\iota: K \rightarrow S^{2} T^{*}$ is injective and that its rank is equal to $m \geq 1$. We choose a fixed complement $T^{\prime}$ of $K$ in $T$ which allows us to split the equation $\operatorname{Bian}(g, R)=0$, for the Riemannian metric $g$, into two pieces. The first one is a first-order equation similar to the equation $\psi_{R}^{\prime}\left(j_{1}(g)\right)=0$ considered above when $R$ is non-degenerate. If $\rho: T^{*} \rightarrow T^{* *}$ is the natural restriction mapping, we denote by $R^{\sharp}$ the inverse of the isomorphism

$$
\rho R^{b}: T^{\prime} \rightarrow T^{\prime *}
$$

If $i: T^{\prime} \rightarrow T$ is the inclusion mapping, we set

$$
S=i R^{\sharp} \rho: T^{*} \rightarrow T .
$$

Then the endomorphism $S R^{b}$ of $T$ is equal to the projection $\pi^{\prime}$ of $T$ onto $T^{\prime}$ corresponding to the decomposition $T=T^{\prime} \oplus K$. We consider the quasi-linear morphism of fibered manifolds

$$
\psi^{\prime \prime}: J_{2}\left(S_{+}^{2} T^{*}\right) \rightarrow T^{*}
$$

determined by

$$
\psi^{\prime \prime}\left(j_{1}(g)\right)=g^{b} \cdot S \cdot \psi_{R}\left(j_{1}(g)\right),
$$

where $g$ is a Riemannian metric on $X$. According to (6), the symbol

$$
\sigma\left(\psi^{\prime \prime}\right): T^{*} \otimes S^{2} T^{*} \rightarrow T^{*}
$$

of $\psi^{\prime \prime}$ is the morphism of vector bundles over $S_{+}^{2} T^{*}$ determined by

$$
\sigma\left(\psi^{\prime \prime}\right)_{g}=-g^{b} \pi^{\prime} g^{\sharp} \sigma\left(B_{g}\right),
$$


where $g$ is a Riemannian metric on $X$; the $l$-th prolongation of the morphism $\sigma\left(\psi^{\prime \prime}\right)_{g}$ will be denoted by $\sigma_{l}\left(\psi^{\prime \prime}\right)_{g}$. The first part of the Bianchi identity is the first-order equation

$$
\psi^{\prime \prime}\left(j_{1}(g)\right)=0 .
$$

To a Riemannian metric $g$ on $X$, we associate the section $\chi(g)$ of $K^{*}$ determined by

$$
\langle\xi, \chi(g)\rangle=\operatorname{Tr}_{g} Q_{\xi},
$$

for $\xi \in K$. The second equation $\chi(g)=0$ arises from the relation (19) and imposes a condition of order zero on the metric $g$. We then attempt to apply the Cartan-Kähler theorem to the system

$$
\operatorname{Ric}(g)=R, \quad j_{1}\left(\psi^{\prime \prime}\left(j_{1}(g)\right)\right)=0, \quad j_{2}(\chi(g))=0
$$

of partial differential equations of order 2 for the metric $g$. This approach fails; indeed, by prolonging the system (27), we find that a set of $m$ additional identities must be taken into consideration. We now derive these scalar-valued identities and then study the new system consisting of (27) and these new identities.

Let $g$ be a Riemannian metric on $X$. The morphism of vector bundles

$$
\sigma(\chi)_{g}: S^{2} T^{*} \rightarrow K^{*}
$$

over $X$ obtained from the symbol $\sigma(\chi)$ of $\chi$ is given by

$$
\left\langle\xi, \sigma(\chi)_{g} h\right\rangle=-\left\langle\xi, \sigma(\chi)_{g} h\right\rangle_{g},
$$

for $h \in S^{2} T^{*}$; its $l$-th prolongation will be denoted by $\sigma_{l}(\chi)_{g}$. We consider the sequence of vector bundles

$$
S^{2} T^{*} \otimes S^{2} T^{*} \stackrel{\sigma_{g}}{\longrightarrow} S^{2} T^{*} \oplus\left(T^{*} \otimes g^{\mathrm{b}}\left(T^{\prime}\right)\right) \oplus\left(S^{2} T^{*} \otimes K^{*}\right) \stackrel{\nu_{g}}{\longrightarrow} K^{*} \rightarrow 0
$$

where the morphism

$$
\sigma_{g}=\sigma(\varphi)_{g} \oplus \sigma_{1}\left(\psi^{\prime \prime}\right)_{g} \oplus \sigma_{2}(\chi)_{g}
$$

is the symbol of the system (27) associated to $g$, and where $\nu_{g}$ is the morphism of vector bundles determined by

$$
\left\langle\xi, \nu_{g}(u \oplus v \oplus w)\right\rangle=\left\langle u+v, Q_{\xi}\right\rangle_{g}-\frac{1}{2} \operatorname{Tr}_{g}\langle\xi, w\rangle,
$$

for $u \in S^{2} T^{*}, v \in T^{*} \otimes g^{b}\left(T^{\prime}\right), w \in S^{2} T^{*} \otimes K^{*}$ and $\xi \in K$.

In $[6, \S 4])$, we first observe that the sequence $(28)$ is a complex, because $K$ is integrable, and then prove: 
Lemma 6. Let $g$ be a Riemannian metric on $X$. If $\chi(g)$ vanishes at $x \in X$ and if $T_{x}^{\prime}$ is the orthogonal complement of $K_{x}$, then the sequence (28) is exact at $x$.

Given the complexity of the morphism $\sigma_{g}$, it is remarkable that the cokernel morphism $\nu_{g}$ can be expressed in such a simple and natural manner. The non-surjectivity of the morphism $\sigma_{g}$ leads us to our additional identities.

Let $x_{0}$ be a point of $X$ and $g_{0}$ be a given Riemannian metric on $X$ satisfying $\operatorname{Tr}_{g} Q_{\xi}=0$, for all $\xi \in K_{x_{0}}$. Since we are interested in the local solvability, we may suppose that $X=\mathbb{R}^{n}$ with a system of linear coordinates $\left(x^{1}, \ldots, x^{n}\right)$ such that $\left\{\partial / \partial x^{1}, \ldots, \partial / \partial x^{m}\right\}$ is a frame for $K$ over $X$. We set $\xi_{j}=\partial / \partial x^{j}$, for $1 \leq j \leq n$. Since $K_{x_{0}}^{\prime \prime}$ contains $K_{x_{0}}$, without loss of generality, we may also suppose that $g_{0, i j}\left(x_{0}\right)=\delta_{i j}$ and that $\left\{\xi_{1}\left(x_{0}\right), \ldots, \xi_{q}\left(x_{0}\right)\right\}$ is a basis for $K_{x_{0}}^{\prime \prime}$, where $q=\nu\left(x_{0}\right) \geq m$. We choose $T^{\prime}$ to be the integrable complement of $K$ in $T$ generated by the vector fields $\left\{\xi_{m+1}, \ldots, \xi_{n}\right\}$ on $X$; thus $T_{x_{0}}^{\prime}$ is the orthogonal complement of $K_{x_{0}}$ in $T_{x_{0}}$ (with respect to $g_{0}$ ). We consider the flat connection $\nabla$ in $T$ for which all the vector fields $\xi_{j}$ are horizontal. This connection preserves $K$ and so, if $g$ is a Riemannian metric on $X$, we may consider the section

$$
P(g)=\nu_{g}\left((\operatorname{Ric}(g)-R) \oplus\left(\nabla \psi^{\prime \prime}(g)\right) \oplus \nabla^{2} \chi(g)\right)
$$

of $K^{*}$. A Riemannian metric $g$, solution of $\operatorname{Ric}(g)=R$, must also satisfy the equation $P(g)=0$, which, in light of the complex (28), is in fact of first order. However, as $P(g)$ arises from the lower order terms of Ric $(g)$, it is an expression which is quadratic in the first derivatives of $g$.

We then consider the new system $M_{2}$ of order 2 consisting of the equations (27) together with the second-order equation $j_{1}(P(g))=0$ for a Riemannian metric $g$. We construct a strongly prolongable 2-jet $j_{2}(g)\left(x_{0}\right)$ of a solution $g$ of the equation $M_{2}$ at $x_{0}$. The main difficulty in proving the existence of such a formal solution at $x_{0}$ consists in finding a 1-jet $j_{1}(g)\left(x_{0}\right)$ of a Riemannian metric $g$ at $x_{0}$, which is a solution of the first-order system

$$
\psi^{\prime \prime}(g)=0, \quad j_{1}(\chi(g))=0, \quad P(g)=0
$$

determined by our system $M_{2}$, such that the symbol of the system $M_{2}$ is involutive at $j_{1}(g)\left(x_{0}\right)$. Then, if $R$ is a real-analytic section, a result of Malgrange [10] asserts that the system $M_{2}$ admits a convergent series solution at $x_{0}$, and thus yields a solution of our original equation. This completes our outline of the proof of Theorem 3 , when $m$ is equal to the rank of $K$ and is $\geq 1$. 


\section{References}

[1] D. Alekseevskij, A. Vinogradov and V. Lychagin, "Basic Ideas and Concepts of Differential Geometry", Encyclopedia of Mathematical Sciences, Vol. 28, Springer-Verlag, Berlin, Heidelberg, New York, 1991.

[2] R. Bryant, S. S. Chern, R. Gardner, H. Goldschmidt and P. Griffiths, "Exterior differential systems", Math. Sci. Res. Inst. Publ., Vol. 18, Springer-Verlag, Berlin, Heidelberg, New York, 1991.

[ 3 ] E. Cartan, "Leçons sur la géométrie des espaces de Riemann", 2nd ed., Gauthier-Villars, Paris, 1946.

[4] D. DeTurck, Existence of metrics with prescribed Ricci curvature: local theory, Invent. Math., 65 (1981), 179-207.

[5] — Metrics with prescribed Ricci curvature, in "Seminar on differential geometry", edited by S. T. Yau, Ann. of Math. Studies, No. 102, Princeton University Press, University of Tokyo Press, Princeton, N.J., 1982, pp. 525-537.

[6 ] D. DeTurck and H. Goldschmidt, Metrics with prescribed curvature of constant rank. I. The integrable case, Adv. in Math., to appear.

[7] J. Gasqui, Sur l'existence locale d'immersions à courbure scalaire donnée, Math. Ann., 219 (1976), 123-126.

[8] H. Goldschmidt, Existence theorems for analytic partial differential equations, Ann. of Math., 86 (1967), 246-270.

[9] Integrability criteria for systems of non-linear partial differential equations, J. Differential Geom., 1 (1967), 269-307.

[10] B. Malgrange, Équations de Lie. II, J. Differential Geom., 7 (1972), 117-141.

[11] J. Milnor, Curvatures of left invariant metrics on Lie groups, Adv. in Math., 21 (1976), 293-329.

D. DeTurk

Department of Mathematics

University of Pennsylvania

Philadelphia, PA 19104

U. S. A.

H. Goldschmidt

Department of Mathematics

Columbia University

New York, NY 10027

U. S. A. 